\title{
Hétérogénéité horizontale des peuplements microphytoplanctoniques et zooplanctoniques en relation avec les paramètres abiotiques dans la retenue El Kansera (Maroc) Horizontal heterogeneity of microphytoplanktonic and zooplanktonic communities in relation to abiotic parameters in the lake-reservoir El Kansera (Morocco)
}

\author{
D. Fqih Berrada, R. Berrada, A. Benzekri et A. Fahde
}

Volume 13, numéro 3, 2000

URI : https://id.erudit.org/iderudit/705392ar

DOI : https://doi.org/10.7202/705392ar

Aller au sommaire du numéro

\section{Éditeur(s)}

Université du Québec - INRS-Eau, Terre et Environnement (INRS-ETE)

\section{ISSN}

0992-7158 (imprimé)

1718-8598 (numérique)

\section{Découvrir la revue}

Citer cet article

Fqih Berrada, D., Berrada, R., Benzekri, A. \& Fahde, A. (2000). Hétérogénéité horizontale des peuplements microphytoplanctoniques et zooplanctoniques en relation avec les paramètres abiotiques dans la retenue El Kansera (Maroc). Revue des sciences de l'eau / Journal of Water Science, 13(3), 213-236. https://doi.org/10.7202/705392ar

\section{Résumé de l'article}

La répartition horizontale du phytoplancton et du zooplancton a été étudiée dans le lac-réservoir El Kansera en période de stabilité thermique, en relation avec certains paramètres abiotiques (transparence, matières en suspension, conductivité, température, oxygène dissous, $\mathrm{pH}$, nutriments azotés et phosphorés).

La répartition longitudinale du phytoplancton montre un gradient décroissant, avec des maxima de biomasse (poids frais) situés au $2 / 3$ de la longueur du réservoir, et des pics de chlorophylle a enregistrés vers l'amont de la retenue. L'abondance du zooplancton suit une évolution quasi-analogue à celle du phytoplancton avec en plus, une répartition hétérogène des Rotifères et des différents stades de développement des Crustacés. Les Rotifères et les petits stades de développement des Cladocères ou des Copépodes sont concentrés dans la zone médiane du lac, tandis que les stades adultes de ces Crustacés dominent vers l'aval. La prédation vertébrée des " Carpes Chinoises ", associée à la mauvaise qualité de la nourriture algale en période d'étiage (blooms à Microcystis), seraient à l'origine de cette répartition. Les résultats de cette étude permettent de distinguer 3 zones de la retenue : la zone d'embouchure de l'oued ${ }^{* *}$ Beht (station $\mathrm{S1}$ ) agitée, turbide et peu oxygènée est la moins productive, la zone médiane (stations S2 et S3), constituant la transition entre le milieu lotique et lacustre, est la plus enrichie en peuplements algaux et la plus peuplée par le zooplancton. Enfin, la zone lacustre proprement dite (stations S4 et S5) est assez productive et constitue un site idéal pour les adultes des Crustacés. 


\title{
Hétérogénéité horizontale des peuplements microphytoplanctoniques et zooplanctoniques en relation avec les paramètres abiotiques dans la retenue El Kansera (Maroc)
}

\author{
Horizontal heterogeneity of microphytoplanktonic \\ and zooplanktonic communities in relation to abiotic \\ parameters in the lake-reservoir El Kansera (Morocco)
}

D. FQIH BERRADA ${ }^{1 *}$, R. BERRADA ${ }^{1}$, A. BENZEKRI ${ }^{2}$, A. FAHDE ${ }^{1}$

Reçu le 30 avril 1999, accepté le $1^{\text {er }}$ septembre $2000^{* *}$.

\section{SUMMARY}

The goal of this study is to assess the degree of horizontal heterogeneity of phytoplankton and zooplankton communities in the lake-reservoir El Kansera (Morocco), in order to define the principal factors responsible for the plankton distribution in the lake.

Five stations have been studied; station $S 1$ is the most influenced by allochthonous inputs from oued Beht (a tributary), stations $S 2$ and $S 3$ represent a transition between the lotic and lacustrine environments, and stations S4 and S5 represent true lacustrine conditions. The phytoplankton and zooplankton samplings were carried out during two seasons in 1996 (spring and summer), with a plankton net which consisted of a silk cone (height: $120 \mathrm{~cm}$, mesh size: $40 \mu \mathrm{m})$. An average sample was prepared by several vertical tows. The samplings of chlorophyll and abiotic parameters were performed at different depths with a polyvinylchloride bottle with a capacity of $5 \mathrm{~L}$ and an automatic closing device.

The phytoplankton counting was done according to the method of Legendre and Watt (1972). The algal biomass $\left(\mu \mathrm{g} \cdot \mathrm{L}^{-1}\right)$ was estimated by chlorophyll-a (method of Lorenzen 1967) and wet weight (method of Lohmann (1908). Zooplankton counting was done in a Dolfuss cell (method of PONT, 1983). The biomass of Rotifers, Cladocerans or Copepods $\left(\mu \mathrm{g} \cdot \mathrm{L}^{-1}\right)$ was calculated according to the method of DUMONT et al. (1975) and BOTTRELL et al. (1976). The physico-chemical analyses were performed at LPEE (Public Laboratory of Essay and Studies). Temperature, $\mathrm{pH}$ and conductivity were measured in

1* Faculté des sciences Aïn Choch de Casablanca (département biologie, BP 5366 Maârif), Casablanca, Maroc.

2. Laboratoire public d'essais et d'études, Centre d'études et de recherche sur l'environnement et la pollution (CEREP), Centre d'activité Oukacha, Bd. Moulay Slimane, Aïn Sebaâ, Casablanca, Maroc.

* Correspondance. E-mail :

** Les commentaires seront reçus jusqu'au 31 octobre 2001. 
situ, with a sounding Horiba U7. Dissolved oxygen was measured by the method of Winkler (RODIER, 1984). Water transparency was measured by Secchi disc (Reynolds). Suspended matter was determined according to the method of Afnor (1994). Nitrates and total phosphorus were analysed by colorimetry, respectively after reduction on a cadmium column and mineralization in acid medium (Afnor, 1994).

The lake-reservoir EI Kansera presents a longitudinal gradient (oued Behtdyke) of biotic and abiotic parameters in relation both to morphometric and hydrologic characteristics and to the importance of allochthonous inputs and the nutrient cycles (nitrates and phosphorus). The maximum phytoplankton abundance (wet weight) is observed at $2 / 3$ of the lake's length, and the chlorophyll- $a$ peaks are recorded in the upper zone. In the spring, two alga species are dominant in the lake, Scenedesmus ecornis and Cyclotella ocellata, whereas in summer, one species is abundant (Microcystis aeruginosa).

Zooplankton abundance shows a similar evolution to that of the phytoplankton, with a longitudinal heterogeneity of Rotifers (Brachionus calyciflorus, Polyarthra vulgaris and Keratella tropica) and different development stages of Cladocerans (Bosmina longirostris and Diaphanosoma brachyurum) and Copepods (Acanthocyclops robustus). Rotifers and the small stages of Crustaceans are concentrated in the median zone, whereas the Crustacean adults are dominant in the deeper point of the reservoir. Vertebrate predation by the "Chinese carp" and the degraded quality of the algae (blooms of Microcystis) also seem to contribute to this distribution.

The results of the study, confirmed by the statistical Student test, indicate three zones in the lake-reservoir: the upper zone (station S1), which is located at the mouth of the oued Beht, is the least_productive due to its shallow depth, low oxygenation, high turbidity and frequent mixing. The intermediate zone, between lotic and lacustrine conditions (stations $\mathrm{S} 2$ and $\mathrm{S3}$ ), is strongly enriched with nutrients (nitrates and phosphorus) and characterised by a higher productivity; this zone presents dense phytoplanktonic populations. The lacustrine zone (stations S4 and S5) is of intermediate productivity, and offers good conditions for large size classes of Crustacea.

Key words: lake-reservoir, horizontal heterogeneity, gradient, oued Beht, inputs, nutrients, nitrates, phosphorus, phytoplankton, biomass, chlorophyll, zooplankton, Rotifers, Cladocerans, Copepods, adults, predation, Microcystis blooms, turbidity.

\section{RÉSUMÉ}

La répartition horizontale du phytoplancton et du zooplancton a été étudiée dans le lac-réservoir El Kansera en période de stabilité thermique, en relation avec certains paramètres abiotiques (transparence, matières en suspension, conductivité, température, oxygène dissous, pH, nutriments azotés et phosphorés).

La répartition longitudinale du phytoplancton montre un gradient décroissant, avec des maxima de biomasse (poids frais) situés au 2/3 de la longueur du réservoir, et des pics de chlorophylle-a enregistrés vers l'amont de la retenue. L'abondance du zooplancton suit une évolution quasi-analogue à celle du phytoplancton avec en plus, une répartition hétérogène des Rotifères et des différents stades de développement des Crustacés. Les Rotifères et les petits stades de développement des Cladocères ou des Copépodes sont concentrés dans la zone médiane du lac, tandis que les stades adultes de ces Crustacés dominent vers l'aval. La prédation vertébrée des « Carpes Chinoises », associée à la mauvaise qualité de la nourriture algale en période d'êtiage (blooms à Microcystis), seraient à l'origine de cette répartition. 
Les résultats de cette étude permettent de distinguer 3 zones de la retenue : la zone d'embouchure de l'oued*** Beht (station S1) agitée, turbide et peu oxygènée est la moins productive, la zone médiane (stations S2 et S3), constituant la transition entre le milieu lotique et lacustre, est la plus enrichie en peuplements algaux et la plus peuplée par le zooplancton. Enfin, la zone lacustre proprement dite (stations S4 et S5) est assez productive et constitue un site idéal pour les adultes des Crustacés.

Mots clés : retenue de barrage, hétérogénéité horizontale, gradient, turbidité, oued $^{* *}$ Beht, phytoplancton, biomasse, chlorophylle, zooplancton, Rotifères, Crustacés, adultes, prédation, blooms à Microcystis.

\section{1 - INTRODUCTION}

Les écosystèmes lacustres, en général, et tout particulièrement les lacs réservoirs présentent fréquemment une répartition spatiale hétérogène et complexe du plancton (THORNTON et al., 1982 ; MATTHEW et JOHN, 1989 ; PINELALLOUL et al., 1989 ; HART, 1990 ; etc.). Les apports des rivières créent des gradients longitudinaux des paramètres abiotiques (matières en suspension, nutriments...) et biotiques (phytoplancton et zooplancton), le long de l'axe rivière-digue. L'amplitude de ces gradients dépend du volume et de la qualité de ces apports, ainsi que de la morphologie du lac (CARMACK et GRAY, 1982 ; JAMES et al., 1987).

En dépit de l'importance écologique de ce phénomène, les études expérimentales relatives à l'occupation spatiale du plancton dans les lac-réservoirs restent limitées. Plusieurs facteurs sont susceptibles de conduire à une répartition hétérogène du phytoplancton ou du zooplancton dans un écosystème lacustre. L'interaction des phénomènes physiques tels la morphologie et la profondeur du lac (GEORGE et EDWARDS, 1976 ; PINEL-ALLOUL et al., 1989), l'advection (GEORGES et EDWARDS, 1976), les conditions héliothermiques (HART, 1990), la teneur en sels nutritifs (MATTHEW et JOHN, 1989), et la turbidité (HART, 1990), et des processus biologiques comme la disponibilité en nourriture (DAGG, 1977), la nage et l'évitement (RINGELBERG, 1969 ; DRENNER et MAC COMAS, 1980), la prédation (KERFOOT, 1975 ; URABE, 1989) et la compétition (DIAMOND, 1979 ; LEVIN, 1984), seraient à l'origine de cette hétérogénéité. Cependant, chaque écosystème lacustre présente ses propres caractéristiques de structure spatiales, qui sont variables en fonction du climat, de la morphométrie, des débits, des apports allochtones, de leur qualité, etc., ce qui nous empêche de généraliser les processus responsables de la répartition spatiale du plancton dans un système lacustre.

Pour ces raisons, une étude de répartition horizontale du phytoplancton et du zooplancton a été entreprise sur la retenue El Kansera (Nord-Ouest du Maroc), lors de deux campagnes saisonnières, dans le but de déterminer d'une part le degré d'hétérogénéité du plancton dans cette retenue et de définir, d'autre part, les principaux facteurs d'ordre abiotique ou biotique responsables de cette répartition. Le but final de cette étude est de montrer l'impact des apports exogènes de l'oued Beht, situé en amont, sur la chaîne trophique de la retenue. 


\section{2 - SITES D'ÉTUDE, MATÉRIEL ET MÉTHODES}

La retenue de barrage El Kansera constitue l'une des plus anciennes retenues du Maroc (mise en eau en 1935). Située au Nord Ouest du pays dans le bassin Beht (figure 1), elle assure le soutien d'étiage et écrête les crues de l'oued Beht, dernier affluent important de l'oued Sebou. Les apports de l'oued Beht sont particulièrement eutrophisants, car ils véhiculent les eaux usées brutes de la ville de Khemisset. Les principales caractéristiques physicochimiques de cet oued, à l'entrée de la retenue, figurent dans le tableau 1. Une dégradation importante de la qualité des eaux du lac-réservoir est ainsi observée depuis la mise en eau du barrage (ONEP, 1996). Cette situation est accentuée par le transit lent de la retenue, où le temps de séjour moyen des eaux est de 15,7 mois (DERRAZ, 1995).
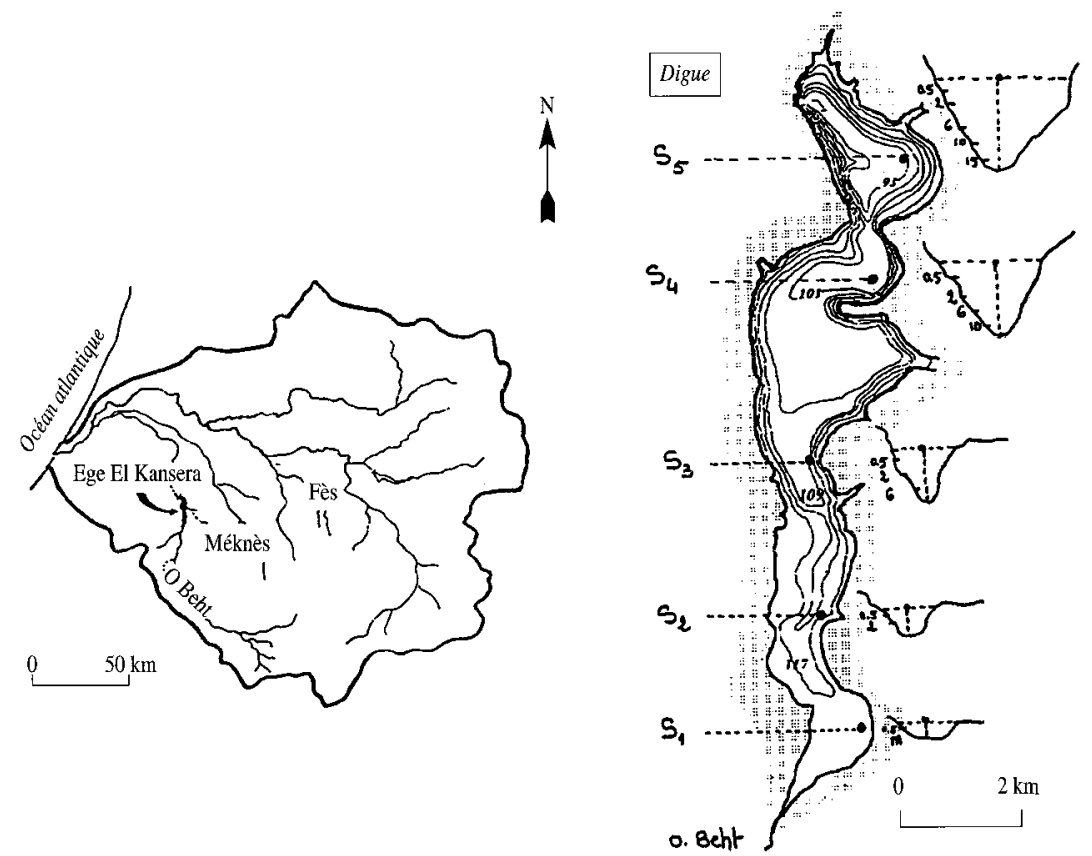

Figure 1 Situation géographique et bathymétrie de la retenue El Kansera et sites d'étude des 5 stations prospectées.

Geographic situation and bathymetry of the lake-reservoir El Kansera and the prospected sites of study.

Le choix des stations à prospecter s'est basé sur la morphologie du lac, le profil bathymétrique, le mode d'alimentation et de gestion et les moyens disponibles. Ainsi, 5 stations ont été prospectées (figure 1) ; la station S1 en amont de la retenue serait soumise aux caprices de l'oued Beht, les stations S2 et S3 représenteraient la transition entre le milieu lotique et le milieu lacustre et les stations $\$ 4$ et $\$ 5$ constitueraient le milieu lacustre proprement dit. L'échantillonnage du phytoplancton et du zooplancton a été réalisé à l'échelle saison- 
nière, plus particulièrement à la fin du printemps 1996 (juin) et en été 1996 (août) lorsque les peuplements planctoniques se développent de façon optimale. Pour comparer les peuplements des différentes stations, nous avons utilisé pour l'échantillonnage le filet à plancton, constitué par un cône de soie à bluter de $120 \mathrm{~cm}$ de haut et de $40 \mu \mathrm{m}$ de vide de maille (le colmatage partiel du filet permet de récolter les algues de taille inférieure). Son diamètre d'embouchure est de $30 \mathrm{~cm}$. Utilisé par des traits verticaux, il parcourt une colonne d'eau de volume calculable, à une vitesse de $0,5 \mathrm{~m} \cdot \mathrm{s}^{-1}$ pour éviter les remous à l'embouchure (LAMOTTE et BOURLIĖRE, 1971 ; LAIR, 1975 ; BRESSAC, 1985). Un échantillon moyen est ainsi constitué par plusieurs traits verticaux, et est fixé immédiatement au formaldéhyde (LAIR, 1975). Le choix de ce filet " passoire " est acceptable dans la mesure où les algues de grosses tailles (Cyanobactéries notamment) ont une très large contribution à la biomasse globale. Les échantillons destinés au dosage de la chlorophylle-a et aux analyses physicochimiques sont prélevés à différentes profondeurs, à l'aide d'une bouteille verticale de type Van Dorn. Les valeurs données pour ces paramètres sont les moyennes sur toute la colonne d'eau.

Les analyses physicochimiques ont été effectuées selon les normes en vigueur, au Laboratoire public d'essais et d'études de Casablanca, agréé par le Comité scientifique français d'accréditation (COFRAC). La température, le $\mathrm{pH}$ et la conductivité ont été mesurés in situ, à l'aide d'une sonde multiparamètre type Horiba U7. L'oxygène dissous a été dosé par la méthode de Winkler (RODIER, 1984). La transparence de l'eau a été évaluée à partir du disque de Secchi (REYNOLDS, 1984). Les matières en suspension ont été déterminées après filtration sur membrane millipore $0,45 \mu$ (Afnor, 1994). Les nitrates ont été dosés par colorimétrie après réduction sur colonne de cadmium, et le phosphore total après minéralisation en milieu acide (Afnor, 1994).

Le comptage des cellules algales a été réalisé au microscope inversé (LEGENDRE et WATT, 1972). La biomasse est exprimée d'une part en chlorophylle-a (LORENZEN, 1967) et d'autre part en poids frais déterminé après estimation du volume des cellules (LOHMANN, 1908). Pour l'espèce Microcystis aeruginosa, le comptage et le biovolume ont été évalués à partir des colonies.

Le dénombrement du zooplancton a été réalisé sous une loupe binoculaire (Olympus $\mathrm{G}^{\star} 40$ ), en cuve Dolfuss. II porte, selon la concentration, soit sur la totalité de l'échantillon, soit sur un sous-échantillon. Le fractionneur utilisé est la boîte de Motoda dont l'erreur de fractionnement est de 8,5 \% (PONT, 1983). Pour le calcul des biomasses, exprimées en poids sec, les Rotifères sont assimilés à des formes géométriques selon la méthode de BOTTRELL et al. (1976), tandis que l'évaluation des biomasses des Cladocères et des Copépodes a été réalisée à l'aide des équations de régression taille-poids établies par DUMONT et al. (1975) et BOTTRELL et al. (1976). Différentes classes de taille ont été déterminées pour ces Crustacés. 
Tableau 1 Évolution des paramètres physicochimiques dans l'oued Beht et dans les 5 stations d'étude, lors des 2 campagnes saisonnières (valeurs moyennes pour la colonne d'eau).

Table 1 Evolution of some physico-chemical parameters in the oued Beht and at the 5 stations of the lake-reservoir, during the two seasonal surveys (mean values for total water column).

\begin{tabular}{|c|c|c|c|c|c|c|c|c|c|c|c|c|}
\hline & \multicolumn{2}{|c|}{ Oued Beht } & \multicolumn{2}{|c|}{ Station \$1 } & \multicolumn{2}{|c|}{ Station S2 } & \multicolumn{2}{|c|}{ Station \$3 } & \multicolumn{2}{|c|}{ Station \$4 } & \multicolumn{2}{|c|}{ Station S5 } \\
\hline & Printemps & Été & Printemps & Été & Printemps & Été & Printemps & Été & Printemps & Été & Printemps & Été \\
\hline $\begin{array}{l}\text { Transparence } \\
\text { (m) }\end{array}$ & 0,05 & 0,1 & 0,2 & 0,5 & 0,4 & 1 & 0,4 & 1 & 0,7 & 1,3 & 0,7 & 1,3 \\
\hline $\begin{array}{c}\text { MES } \\
(\mathrm{mg} / \mathrm{l})\end{array}$ & 5237 & 78 & 5000 & 59 & 1500 & 25 & 900 & 10 & 500 & 4 & 150 & 4 \\
\hline $\begin{array}{l}\text { Conductivité } \\
(\mu \mathrm{S} / \mathrm{cm})\end{array}$ & 854 & 981 & 941 & 840 & 942 & 785 & 707 & 640 & 640 & 620 & 588 & 600 \\
\hline $\begin{array}{c}\text { Température } \\
\left({ }^{\circ} \mathrm{C}\right)\end{array}$ & 26,4 & 27 & 28,6 & 29,6 & 27,5 & 28,5 & 26,8 & 27,2 & 23,7 & 24,3 & 22,5 & 23,8 \\
\hline $\mathrm{pH}$ & 8 & 8,4 & 8,2 & 8,3 & 8,2 & 8,3 & 8,2 & 8,3 & 8,2 & 8,2 & 8 & 8,1 \\
\hline $\begin{array}{c}\text { Oxygène } \\
\text { dissous (mg/l) }\end{array}$ & 7,2 & 9 & 5,5 & 6 & 8,8 & 8,9 & 9,5 & 8,5 & 7 & 4,9 & 6,7 & 4,5 \\
\hline 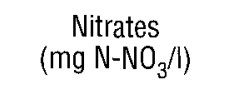 & 1,9 & 4 & 5 & 4,1 & 8 & 4,2 & 9,8 & 5 & 10,5 & 6 & 10,7 & 6,8 \\
\hline $\begin{array}{l}\text { Phosphore } \\
(\mu \mathrm{g} \text { PT/I) }\end{array}$ & 600 & 186 & 400 & 372 & 250 & 280 & 120 & 200 & 78 & 130 & 57 & 119 \\
\hline $\begin{array}{l}\text { Matière organique } \\
\qquad\left(\mathrm{mg} \mathrm{O}_{2} / \mathrm{l}\right)\end{array}$ & 13,1 & 12 & 10 & 9,5 & 6,2 & 6 & 5,8 & 5,7 & 4 & 3,5 & 2,1 & 3 \\
\hline
\end{tabular}




\section{3 - RÉSULTATS}

\subsection{Facteurs abiotiques}

Le tableau 1 montre les valeurs des paramètres abiotiques durant les deux saisons d'étude, dans les 5 stations prospectées et dans l'oued Beht.

L'évolution horizontale de la transparence de l'eau montre un gradient longitudinal croissant de l'amont à l'aval de la retenue, avec des valeurs oscillant entre un minimum de $0,2 \mathrm{~m}$ à $\mathrm{S} 1$ et un maximum de $1,3 \mathrm{~m}$ à $\mathrm{S} 5$. Cette évolution serait liée au gradient longitudinal décroissant des matières en suspension, dont les teneurs varient de $5000 \mathrm{mg} / \mathrm{l}$ dans la station S1 à $4 \mathrm{mg} / \mathrm{l}$ dans la station S5. Les valeurs élevées en saison printanière sont dues aux apports allochtones de l'oued Beht, fortement chargés en matières en suspension (crue printanière). L'évolution de la conductivité montre par ailleurs un gradient décroissant, indiquant que les eaux sont de moins en moins minéralisées à mesure que l'on s'éloigne de l'oued Beht. La température de l'eau varie dans toutes les stations entre 22,5 et $28,6{ }^{\circ} \mathrm{C}$ au printemps et entre 23,8 et $29,6{ }^{\circ} \mathrm{C}$ en été. Les teneurs en oxygène dissous oscillent entre 6,7 et $9,5 \mathrm{mgO}_{2} / \mathrm{l}$ au printemps et entre 4,5 et $9 \mathrm{mgO}_{2} / 1$ en été. Ces deux paramètres indiquent de plus, un gradient vertical bien marqué en saison estivale (tableau 2) ; les coefficients de variation les plus élevés se trouvent dans les couches superficielles, tandis qu'ils sont les plus faibles en profondeur. Ces résultats seraient liés au phénomène de stratification thermique estivale qui caractérise la retenue. $\mathrm{Le} \mathrm{pH}$ est alcalin dans toutes les stations, et oscille entre 8 et 8,2 au printemps et entre 8,1 et 8,3 en été.

Tableau 2 Variabilité estivale verticale de la température et de l'oxygène dissous aux différentes stations de la retenue.

Table 2 Vertical variability of temperature and dissolved oxygen at different stations of the lake-reservoir during the summer.

\begin{tabular}{|c|c|c|c|c|c|c|c|c|}
\hline & \multicolumn{4}{|c|}{ Température $\left({ }^{\circ} \mathrm{C}\right)$} & \multicolumn{4}{|c|}{ Oxygène dissous (mg $\mathrm{O}_{2} / \mathrm{l}$ ) } \\
\hline & $\mathbf{n}$ & $\overline{\mathbf{X}}$ & SD & CV $(\%)$ & n & $\overline{\bar{X}^{\prime}}$ & SD & CV (\%) \\
\hline 0,5 & 5 & 27,6 & 1,2 & 4,3 & 5 & 9,3 & 1,7 & 18,2 \\
\hline 2 & 4 & 26 & 0,7 & 2,7 & 4 & 8,6 & 1,4 & 16,6 \\
\hline 6 & 3 & 24,6 & 0,2 & 0,8 & 3 & 8,8 & 0,5 & 5,7 \\
\hline 10 & 2 & 19,4 & 0 & 0 & 2 & 5,2 & 0,6 & 11,5 \\
\hline 15 & 1 & $(19)^{*}$ & - & - & 1 & $(0,6)^{*}$ & - & 一 \\
\hline
\end{tabular}

où $n=$ nombre d'échantillons

$\overline{\mathrm{X}}=$ moyenne de la température $\left({ }^{\circ} \mathrm{C}\right)$

$\bar{X}^{\prime}=$ moyenne de l'oxygène dissous $(\mathrm{mg} / \mathrm{l})$

$\mathrm{SD}=$ écarts types

$\mathrm{CV}=$ coefficients de variation

$\left({ }^{*}\right)=$ valeur d'un seul dosage $(\mathrm{à}-15 \mathrm{~m})$

L'évolution des teneurs en nitrates le long de l'axe amont-aval montre un léger gradient croissant, avec des valeurs oscillant entre un minimum de $4 \mathrm{mg} / \mathrm{l}$ 
à S1 et un maximum de $10,7 \mathrm{mg} / \mathrm{l}$ à S5. À l'inverse, l'évolution du phosphore total suit un gradient décroissant avec un maximum de $400 \mu \mathrm{g} / \mathrm{l}$ à $\mathrm{S} 1$ et un minimum de $57 \mu \mathrm{g} / \mathrm{l}$ à S5. Les apports de l'oued Beht, particulièrement riches en cet élément ( $600 \mu \mathrm{gPT} / \mathrm{l}$ lors de la crue printanière), contribuent à l'enrichissement en phosphore de la zone " amont ", tandis que la zone " aval " est moins affectée par la crue du fait de la sédimentation croissante de cet élément le long de l'axe oued Beht-digue. Toutefois, en saison estivale l'anoxie qui règne en profondeur dans les stations S4 et S5 entraîne la réduction du phosphore et sa libération sur toute la colonne d'eau (figure 2) : ce phénomène de relargage est observé dans d'autres retenues marocaines (DAFIR, 1997).
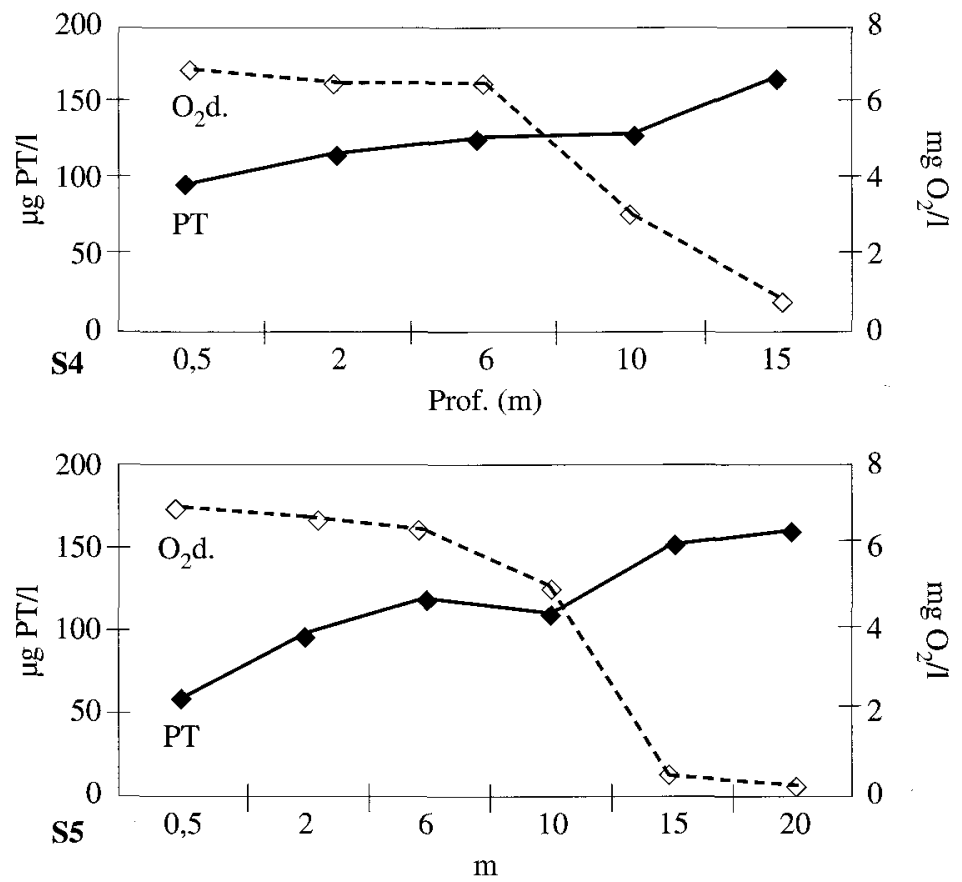

Figure 2 Évolution verticale du phosphore total (PT) et de l'oxygène dissous $\left(\mathrm{O}_{2} \mathrm{~d}\right)$ aux stations $\mathrm{S} 4$ et $\mathrm{S} 5$ durant la campagne estivale.

Vertical evolution of total phosphorus (PT) and dissolved oxygen $\left(\mathrm{O}_{2} \mathrm{~d}\right)$ at stations S4 and S5 during the estival campaigne.

\subsection{Répartition longitudinale du phytoplancton}

\subsubsection{Campagne printanière}

L'étude qualitative du phytoplancton en cette saison montre une composition spécifique assez variée et similaire dans chacune des stations étudiées. Tous les groupes algaux sont bien représentés, en particulier les Diatomées Cyclotella ocellata et Aulacoseira granulata et les Chlorococcales Scenedesmus ecornis et Pediastrum simplex. Les Volvoccales et les Desmidiales sont relativement rares ou n'ont pas pu être échantillonnés avec le filet à grosses mailles. 
L'évolution longitudinale de la chlorophylle-a montre un gradient décroissant, les concentrations maximales se situant au niveau de la station $\mathrm{S} 2$ avec une teneur moyenne sur toute la colonne d'eau de $8,3 \mu \mathrm{g} / \mathrm{l}$ (figure 3). L'évolution verticale de la chlorophylle dans les différentes stations montre par ailleurs, des variations relativement grandes entre stations et aux mêmes profondeurs (tableau 3). Toutefois, les valeurs de ces coefficients semblent assez proches pour différentes profondeurs, ce qui indiquerait que la chlorophylle-a varie plus en cette saison suivant un gradient longitudinal que vertical.

Tableau 3 Variabilité verticale de la chlorophylle-a dans les différentes stations de la retenue.

Table 3 Vertical variability of chlorophyll-a at different stations of the lakereservoir.

\begin{tabular}{|c|c|c|c|c|c|c|c|c|}
\hline \multirow{2}{*}{} & \multicolumn{4}{|c|}{ Printemps (06/1996) } & \multicolumn{4}{c|}{ Été (08/1996) } \\
\cline { 2 - 9 } & $\mathbf{n}$ & $\overline{\mathbf{X}}$ & $\mathbf{S D}$ & $\mathbf{C V}(\%)$ & $\mathbf{n}$ & $\overline{\mathbf{X}}$ & $\mathbf{S D}$ & $\mathbf{C V}(\%)$ \\
\hline & 5 & 7,3 & 1,4 & 19,7 & 5 & 12,4 & 10,3 & 82,7 \\
2 & 4 & 5,9 & 1,4 & 23,1 & 4 & 9,3 & 4,9 & 53,1 \\
6 & 3 & 5,3 & 1,5 & 28,3 & 3 & 6,6 & 0,6 & 9,1 \\
10 & 2 & 1,9 & 0,6 & 33,3 & 2 & 4,3 & 1,4 & 33,3 \\
15 & 1 & $(1,8)^{*}$ & - & - & 1 & $(1,9)^{*}$ & - & - \\
\hline
\end{tabular}

où $\mathrm{n}=$ nombre d'échantillons

$\bar{X}=$ moyenne de la température $\left({ }^{\circ} \mathrm{C}\right)$

$\bar{X}^{\prime}=$ moyenne de l'oxygène dissous $(\mathrm{mg} / \mathrm{l})$

$\mathrm{SD}=$ écarts types

$\mathrm{CV}=$ coefficients de variation

$\left({ }^{*}\right)=$ valeur d'un seul dosage $(\grave{a}-15 \mathrm{~m})$

La répartition horizontale de la biomasse algale (poids frais) indique une évolution similaire à celle de la chlorophylle, avec un pic à S2 de l'ordre de $26,5 \mathrm{mg} / \mathrm{l}$ (figure 3). Ce pic est représenté en bonne partie par les espèces $S$. ecornis et $C$. ocellata.

\subsubsection{Campagne estivale}

Comme pour la saison précédente, la composition spécifique algale est similaire dans les 5 stations. Différents groupes algaux sont représentés, en particulier les Cyanobactéries Microcystis aeruginosa et Oscillatoria tenuis, la Dinophycée Peridiniopsis elpatiweskii, la Desmidiale Staurastrum sp., et la Chlorococcale Oocystis crassa. L'exception est toutefois observée dans la station S1, qui renferme deux espèces particulières Trachelomonas lefevri et Gyrosigma acuminatum.

L'évolution longitudinale de la chlorophylle-a montre un gradient décroissant, avec un maximum à S1 de l'ordre de $32 \mu \mathrm{g} / \mathrm{l}$ (figure 4). De plus, l'évolution verticale dans les différentes stations montre de grandes variations entre stations aux mêmes profondeurs (tableau 3). Les coefficients de variation les plus élevés se trouvent à $-0,5$ et $-2 \mathrm{~m}$ (respectivement 82,7 et $53 \%$ ) tandis qu'ils sont plus faibles en profondeur. Ces variations peuvent être expliquées par la 

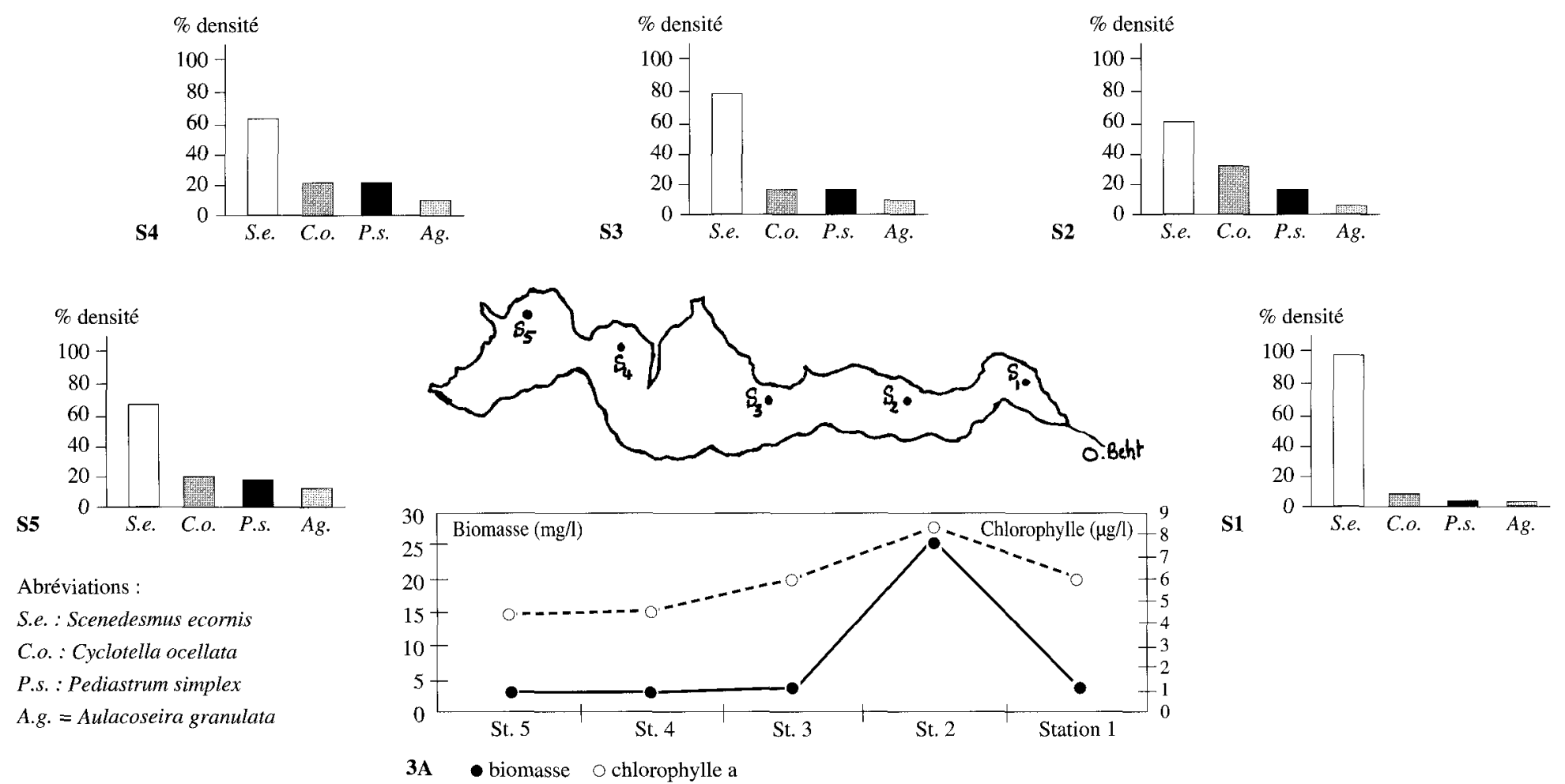

\section{Abréviations}

S.e. : Scenedesmus ecornis

C.o. : Cyclotella ocellata

P.s. : Pediastrum simplex

A.g. = Aulacoseira granulata

3A biomasse $O$ chlorophylle a

Figure 3 Distribution longitudinale de la biomasse algale (poids frais) et de la chlorophylle a (3A) et des \% de densité des principales espèces dans les différentes stations de la retenue, durant la campagne printanière (valeurs moyennes de la colonne d'eau).

Longitudinal distribution of alga biomass (wet weight) and clorophyll a (3A) and \% of density of principal species at different stations of the lake, during the spring campaigne (mean values on total water column). 
répartition inégale du phytoplancton dans la colonne d'eau en cette saison. Ainsi, la chlorophylle-a varierait en été en fonction du gradient horizontal et vertical.

L'évolution longitudinale de la biomasse algale (poids frais) montre des valeurs maximales situées entre les stations S2 et S3 avec respectivement 320 et $361 \mathrm{mg} / \mathrm{l}$ (figure 4). Ces biomasses élevées sont en relation avec la prolifération de la Cyanobactérie $M$. aeruginosa. En dépit de l'abondance d'autres espèces algales dans cette zone comme $P$. elpatiweskii, C. ocellata, O. crassa, ou $O$. tenuis, le pic de biomasse est imputable à la Cyanobactérie du fait du biovolume largement plus important de cette forme coloniale.

\subsection{Répartition longitudinale du zooplancton}

\subsubsection{Campagne printanière}

La composition spécifique du zooplancton est quasi-analogue dans les différentes stations. Les trois groupes zooplanctoniques sont représentés. Chez les Rotifères, plusieurs espèces se développent, en particulier Polyarthra vulgaris, Brachionus calyciflorus et Keratella tropica. Chez les Cladocères, Bosmina longirostris et Diaphanosoma brachyurum sont très communs, alors que chez les Copépodes une espèce est fortement dominante (Acanthocyclops robustus).

L'évolution longitudinale des effectifs zooplanctoniques et de leurs biomasses (figure 5) présente un pic au niveau de la station S3 (6 575 individus/l soit $1996 \mu \mathrm{g} / \mathrm{l})$. En termes d'effectifs, ce pic est représenté à $70 \%$ par les Nauplii larves de Copépodes, à $15 \%$ par les Rotifères, à $7 \%$ par $D$. brachyurum, à $6 \%$ par $B$. longirostris et à $2 \%$ par $A$. robustus. En termes de biomasses, compte tenu de la disparité des tailles, il est constitué à seulement $38 \%$ par les larves de Copépodes, à $28 \%$ par $D$. brachyurum, à $16,6 \%$ par $A$. robustus et à $14 \%$ par $B$. longirostris.

On constate aussi une opposition amont-aval avec une concentration des Rotifères et des jeunes stades de Cladocères et de Copépodes aux stations S1, S2 et même S3 et, en général une dominance des stades adultes des Crustacés aux stations S4 et S5 (figure 6). Ainsi pour A. robustus, le taux maximal des adultes atteint $94 \%$ à la station S5 avec $30 \%$ de mâles et $66 \%$ de femelles (dont $20 \%$ sont ovigères). De même pour $D$. brachyurum, ce taux est maximal à la station S5 (63\%) avec $15 \%$ de femelles ovigères. En revanche chez $B$. longirostris, il se situe à la station $\$ 3(63 \%)$ avec $18 \%$ de femelles ovigères. Chez les Rotifères (tableau 4), le taux maximal de femelles ovigères est en revanche enregistré à la station $\mathrm{S} 3$, avec $80 \%$ chez B. calyciflorus, $55 \%$ chez $K$. tropica et $45 \%$ chez P. vulgaris.

\subsubsection{Campagne estivale}

La répartition et la composition spécifique du peuplement zooplanctonique estival sont similaires à celles observées au cours de la saison précédente. Seule l'espèce $B$. longirostris fait défaut en été, ce qui s'explique par ses préférences pour les températures printanières (KWIK et CARTER, 1975).

Les effectifs zooplanctoniques et leurs biomasses respectives (figure 7 ) présentent un pic au niveau de la station S3 (6066 individus/l soit $8037 \mu \mathrm{g} / \mathrm{l})$. Ce 

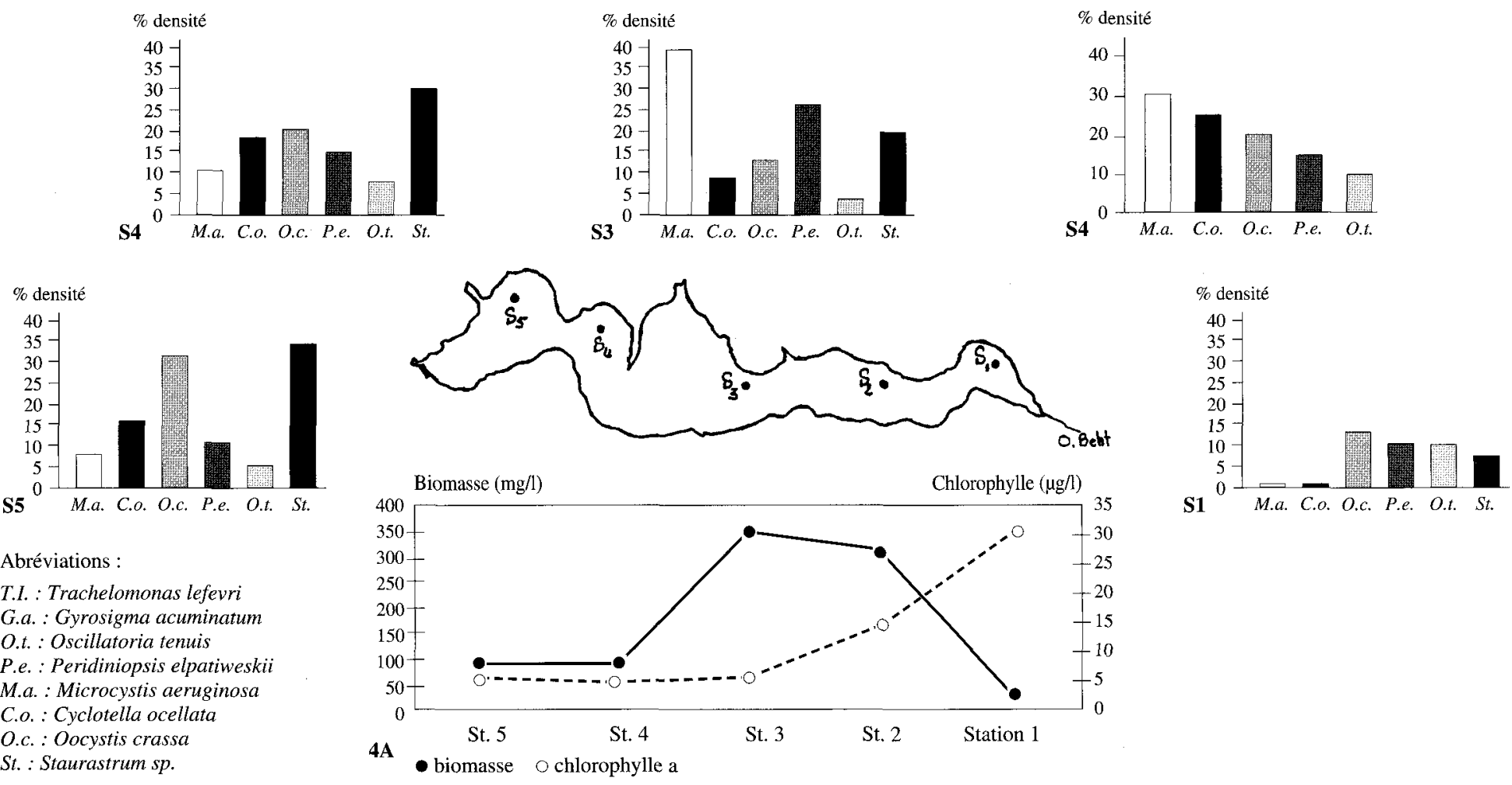

Abréviations :

T.I. : Trachelomonas lefevri

G.a. : Gyrosigma acuminatum

O.t. : Oscillatoria tenuis

P.e. : Peridiniopsis elpatiweskii

M.a. : Microcystis aeruginosa

C.o. : Cyclotella ocellata

O.c. : Oocystis crassa

St. : Staurastrum sp.

Figure 4 Distribution longitudinale de la biomasse algale (poids frais) et de la chlorophylle a (4A) et des \% de densité des principales espèces dans les différentes stations de la retenue, durant la campagne estivale (valeurs moyennes de la colonne d'eau).

Longitudinal distribution of alga biomass (wet weight) and clorophyll a (4A) and \% of density of principal species at different stations of the lake, during the summer campaigne (mean values on total water column). 
pic est dominé, en densité, par le Rotifère $B$. calyciflorus (42\%), le Copépode $A$. robustus $(25,8 \%$ ) et le Cladocère $D$. brachyurum (22 \%). Mais en biomasse, il est dominé par les Crustacés.

Comme pour la campagne précédente, une concentration de Rotifères est observée aux stations S1, S2 et S3, alors que les Crustacés sont concentrés aux stations $\mathrm{S} 4$ et $\mathrm{S} 5$ avec une dominance de leurs stades adultes à ce niveau (figure 8). Ainsi chez A. robustus, $79 \%$ de l'effectif du Copépode ont atteint le stade adulte à la station S5 avec $39 \%$ de mâles et $40 \%$ de femelles (dont $12 \%$ sont ovigères). De même, à cette station chez $D$. brachyurum, $75 \%$ des effectifs sont des adultes et comptent $22 \%$ de femelles ovigères. En revanche, chez les Rotifères (tableau 4), le taux maximal de femelles ovigères est enregistré soit en S2 (avec $60 \%$ chez $K$. tropica), soit en S3 (avec $60 \%$ chez B. calyciflorus et $52 \%$ chez $P$. vulgaris).

Tableau 4 Effectifs de trois espèces de Rotifères (Brachionus calyciflorus, Polyarthra vulgaris et Keratella tropica) aux différentes stations de la retenue, durant les 2 campagnes de prélèvement, et \% de densité des femelles ovigères $(Q w)$ de ces espèces.

Table 4 Density of three species of Rotifers (Brachionus calyciflorus, Polyarthra vulgaris and Keratella tropica) at different stations of the lakereservoir, during the two surveys, and \% abundance of gravid females $(Q w)$ of these species.

\begin{tabular}{|c|c|c|c|c|c|c|}
\hline & \multicolumn{2}{|c|}{ B. calyciflorus } & \multicolumn{2}{|c|}{ P. vulgaris } & \multicolumn{2}{|c|}{ K. tropica } \\
\hline & Printemps & Été & Printemps & Été & Printemps & Été \\
\hline $\begin{array}{l}\text { S1 } \\
\text { effectif total } \\
\text { (ind./I) }\end{array}$ & 10 & 50 & 6 & 10 & 3 & 20 \\
\hline \% Qw & 0 & 0 & 0 & 0 & 0 & 0 \\
\hline $\begin{array}{l}\text { S2 } \\
\text { effectif total } \\
\text { (ind./I) }\end{array}$ & 322 & 1300 & 242 & 242 & 85 & 2125 \\
\hline \% Qw & 86 & 45 & 30 & 38 & 31 & 60 \\
\hline $\begin{array}{l}\text { S3 } \\
\text { effectif total } \\
\text { (ind./l) }\end{array}$ & 366 & 2590 & 328 & 150 & 62 & 334 \\
\hline$\%$ & 80 & 60 & 45 & 52 & 55 & 48 \\
\hline $\begin{array}{l}\text { S4 } \\
\text { effectif total } \\
\text { (ind./l) }\end{array}$ & 50 & 28 & 35 & 140 & 15 & 168 \\
\hline \% Qw & 45 & 30 & 30 & 41 & 10 & 40 \\
\hline $\begin{array}{l}\text { S5 } \\
\text { effectif total } \\
\text { (ind./l) }\end{array}$ & 48 & 22 & 34 & 22 & 16 & 178 \\
\hline \% QW & 39 & 33 & 28 & 38 & 8 & 35 \\
\hline
\end{tabular}



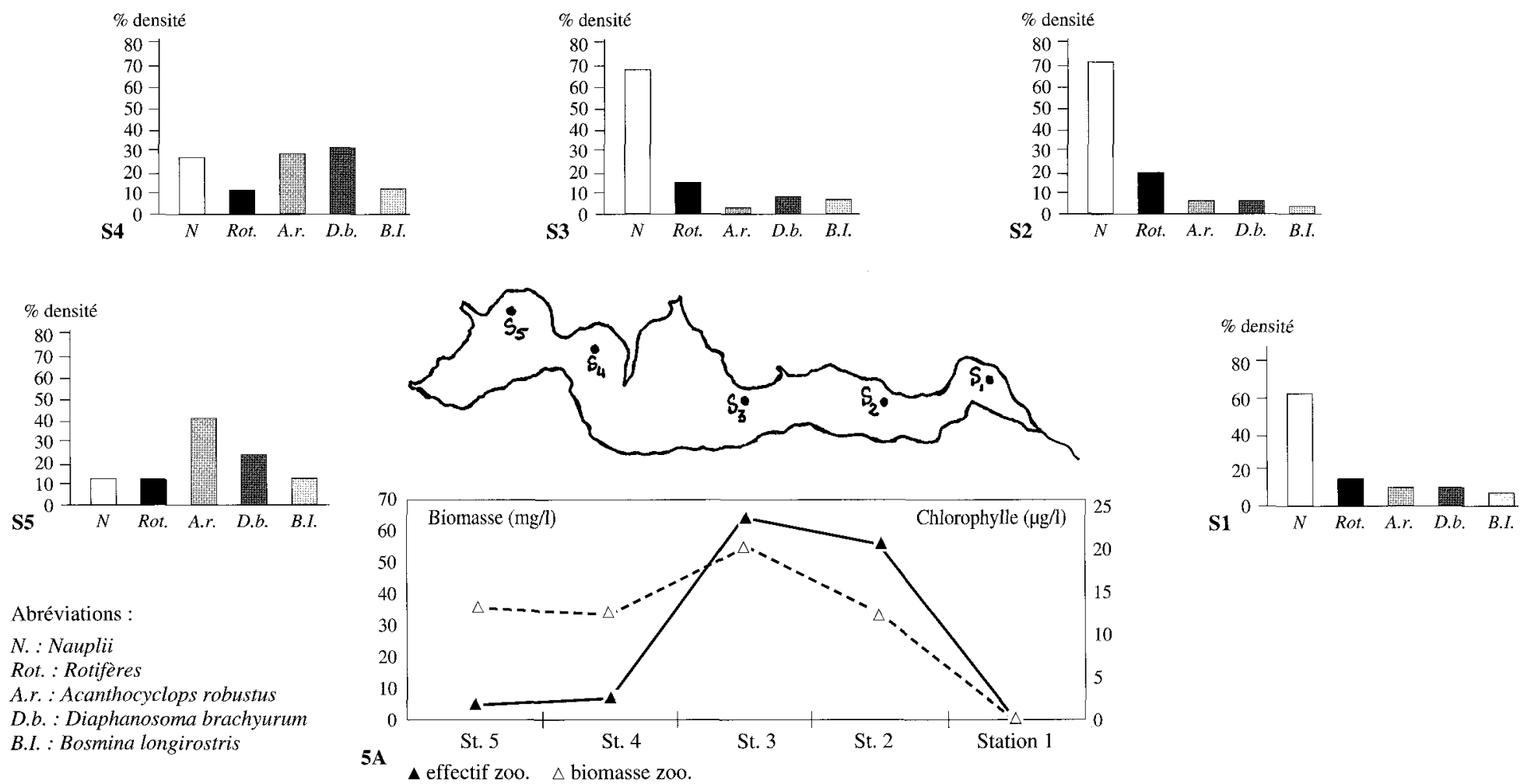

Abréviations :

N. : Nauplii

Rot.: Rotifères

A.r. : Acanthocyclops robustus

D.b. : Diaphanosoma brachyurum

B.I. : Bosmina longirostris

5A $\triangle$ effectif zoo. $\triangle$ biomasse zoo.

Figure 5 Distribution longitudinale de la biomasse zooplanctonique $(5 \mathrm{~A})$ et des $\%$ de densité des principales espèces dans les différentes stations de la retenue, durant la campagne printanière (valeurs moyennes de la colonne d'eau).

Longitudinal distribution of zooplanctonic density and biomass $(5 \mathrm{~A})$ and $\%$ of density of principal species at different stations of the lake, during the spring campaigne (mean values on total water column). 


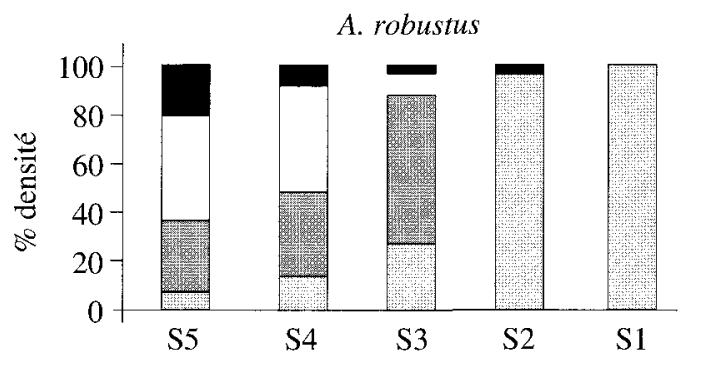

copépodites mâles $\square$ femelles $\mathbf{\square}$ f. ovigères
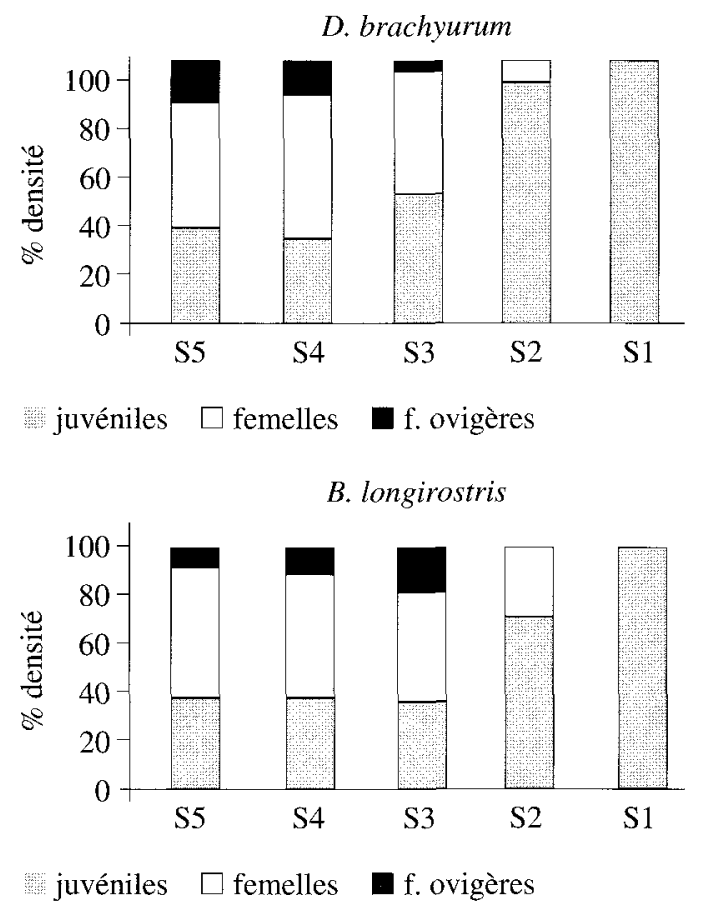

Figure 6 Distribution longitudinale des différents stades de développement du Copépode Acanthocyclops robustus et des Cladocères Diaphanosoma brachyurum et Bosmina longirostris dans la retenue, durant la campagne printanière (en \% de densité).

Longitudinal distribution of different development's stages of the Copepod Acanthocyclops robustus and Cladocers Diaphanosoma brachyurum and Bosmina longirostris, during the spring campaigne (in \% of density)

\section{4 - DISCUSSION}

L'abondance des espèces phytoplanctoniques et zooplanctoniques varie de façon significative de l'amont à l'aval, les maxima survenant en général 

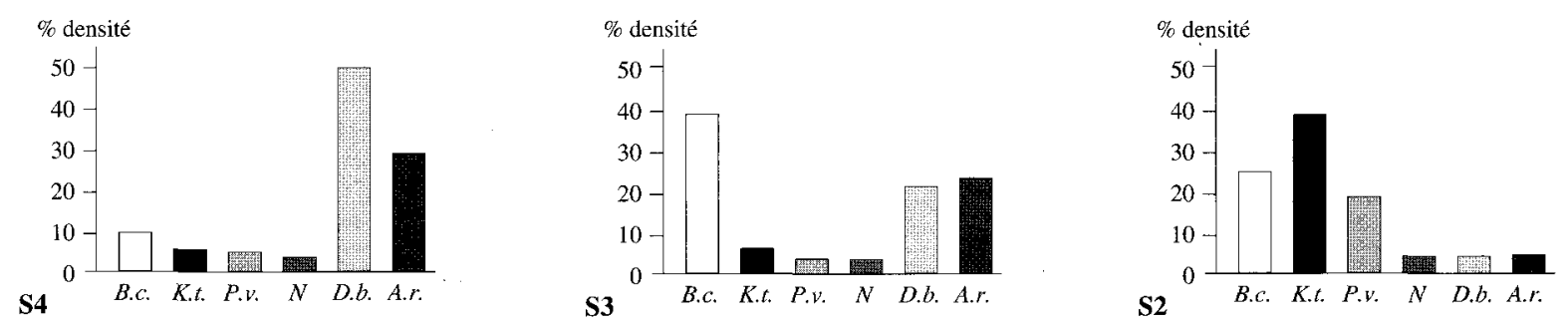

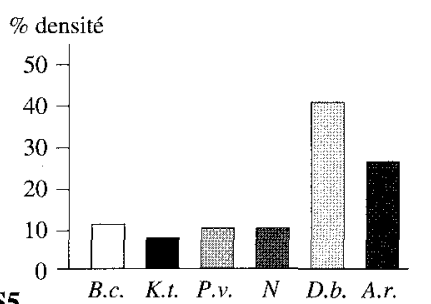

Abréviations :

B.c. : Brachionus calyciflorus

K.t. : Keratella tropica

P.v. : Polyartha vulgaris

N: Nauplii

D.b. Diaphanosoma brachyurum

A.r. : Acanthocyclops robustus
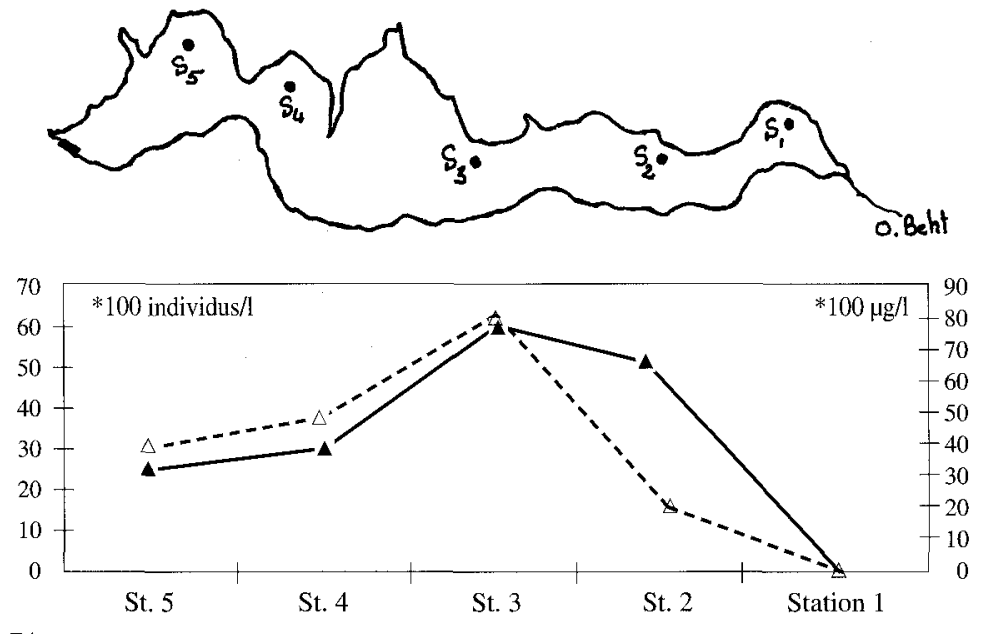

$\%$ densité

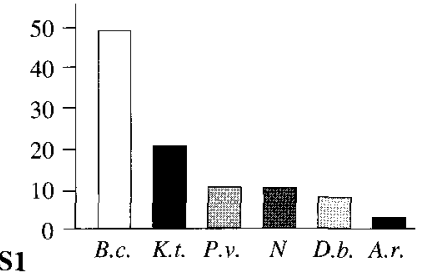

7A $\triangle$ effectif zoo. $\triangle$ biomasse zoo.

Figure 7 Distribution longitudinale de l'effectif de la biomasse zooplanctonique $(7 \mathrm{~A})$ et des $\%$ de densité des principales espèces dans les différentes stations de la retenue, durant la campagne estivale (valeurs moyennes de la colonne d'eau).

Longitudinal distribution of zooplanctonic density and biomass $(7 A)$ and $\%$ of density of principal species at different stations of the lake, during the summer campaigne (mean values on total water column). 


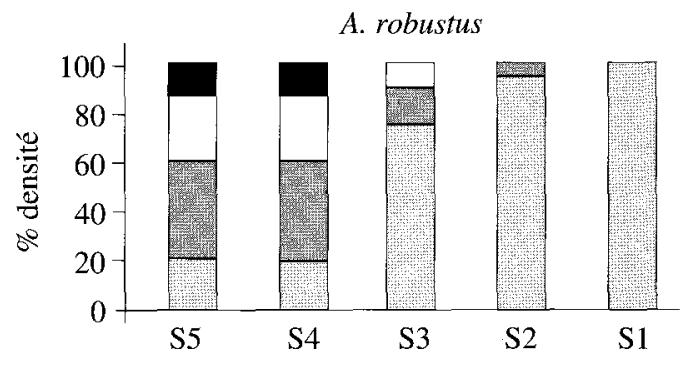

copépodites 险 mâles $\square$ femelles $\square$ f. ovigères

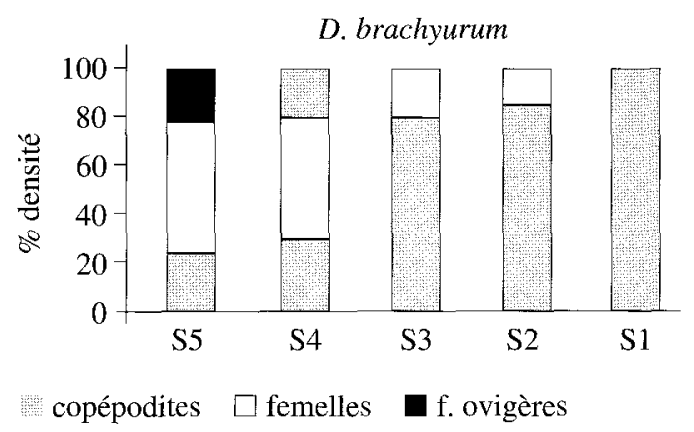

Figure 8 Distribution longitudinale des différents stades de développement $\mathrm{du}$ Copépode Acanthocyclops robustus et du Cladocère Diaphanosoma brachyurum.

Longitudinal distribution of different development's stages of Copepod Acanthocyclops robustus and Cladocer Diaphanosoma brachyurum, during the summer campaigne (in \% of density).

entre les stations S2 et S3, soit environ au 2/3 de la longueur du réservoir. Les apports allochtones de l'oued Beht, chargés en matières en suspension et en éléments nutritifs, interfèrent dans la régulation de cette abondance. Ainsi les stations S2 et S3, qui constituent la transition entre la milieu lotique et le milieu lacustre, autorisent un bon développement du phytoplancton. Cela s'explique par le fait qu'elles offrent une bonne transparence et une bonne minéralisation et qu'elles sont, de plus, chauffées et oxygénées sur toute la colonne d'eau du fait de leur faible profondeur. Enfin, ces stations sont fortement enrichies en éléments nutritifs azotés mais surtout phosphorés.

- Au printemps, les teneurs en chlorophylle-a sont maximales dans cette zone et quasi-homogènes sur toute la colonne d'eau, témoignant d'une forte activité photosynthétique ( $\mathrm{pH}$ alcalin et teneurs élevées en oxygène dissous). Deux espèces algales sont particulièrement stimulées par ces conditions; S. ecornis et C. ocellata. Selon GOLTERMAN (1973), LE COHU (1974) et LOUDIKI (1990), ces espèces sont très dépendantes de la disponibilité des nutriments (azotés et phosphorés) dans le milieu.

- En été, la Cyanobactérie $M$. aeruginosa est nettement dominante dans cette zone, formant des tapis étalés à la surface de l'eau. Les teneurs en chlorophylle-a sont relativement élevées et homogènes dans toute la colonne 
d'eau, mais restent inférieures à celles de la station $\mathbf{S 1}$. Selon plusieurs auteurs (ZOHARY et ROBARTS, 1989 ; KÖHLER, 1992), les cellules algales de Microcystis diminuent leurs contenus cellulaires en chlorophylle pour s'adapter aux fortes radiations lumineuses de la saison d'été. De plus, la faible transparence de l'eau dans la station S1 (zone d'embouchure) entraîne une diminution de la pénétration de la lumière dans la couche d'eau, ce qui conduirait les cellules algales à une synthèse accrue de la chlorophylle pour utiliser au mieux l'énergie lumineuse disponible (MATTHEW et JOHN, 1989 ; MALKI, 1994). La prolifération de $M$. aeruginosa dans les stations $S 2$ et S3 semble favorisée par les fortes teneurs en phosphore (KÖHLER, 1992 ; VARIS, 1993 ; DERRAZ, 1995). Or, la faible profondeur de cette zone, associée aux vents quasi-permanents qui règnent au niveau de la retenue (Chergui), favorisent la resuspension des sédiments fortement enrichis en cet élément (DERRAZ, 1995 ; ABDALLAOUl et al., 1998). De ce fait, les faibles débits de l'oued Beht en cette période d'étiage sont compensés par les apports internes en phosphore. L'absence de $M$. aeruginosa dans la station $\mathrm{S} 1$ serait en relation avec la faible transparence de cette zone qui limite le taux de pénétration de la lumière dans la couche d'eau. La rareté locale de $M$. aeruginosa serait alors surtout la conséquence des turbulences de cette zone. Selon KÖHLER (1992), VARIS (1993) et DERRAZ (1995), le développement de cette Cyanobactérie placée sous des conditions nutritives non limitantes n'est que fonction de l'éclairement et de la température. En revanche, la rareté de cette espèce aux stations S4 et S5 tiendrait au fait que les colonies flottantes de Microcystis sont facilement entraînées par les vents de direction Est (Chergui) vers la zone «amont " de la retenue, où elles tendent à se concentrer. L'effet du broutage par le zooplancton est exclu, du fait du caractère toxique de l'algue (ZHENRONG, et al. 1992 ; RFINIKAINEN et al., 1994 ; THÉBAULT et al., 1995 ; CHRISTOFFERSEN, 1996 ; ENRISKEN et MOESTRYP, 1997).

Ainsi, 3 zones du lac-réservoir ont pu être distinguées : la zone d'embouchure de l'oued Beht (S1) la moins productive, la zone médiane (S2-S3) la plus riche en biomasse algale et la plus productive, et la zone lacustre (S4-S5) moins riche en phytoplancton et moyennement productive. La comparaison des biomasses algales moyennes aux 5 stations, à l'aide du test de Student, confirme l'ensemble de ces constatations (tableau 5).

Tableau 5 Comparaison des biomasses phytoplanctoniques moyennes $(\mathrm{tm})$ et des biomasses zooplanctoniques moyennes ( $t$ ' $m$ ) entre les différentes stations, à l'aide du test statistique de Student.

Table 5 Comparison of phytoplanktonic biomass $(\mathrm{tm})$ and zooplanktonic biomass (t'm) between different stations, with the Student statistical.

\begin{tabular}{|c|c|c|c|c|c|c|c|c|c|c|}
\hline Stations & $\mathbf{1 - 2}$ & $\mathbf{1 - 3}$ & $\mathbf{1 - 4}$ & $\mathbf{1 - 5}$ & $\mathbf{2 - 3}$ & $\mathbf{2 - 4}$ & $\mathbf{2 - 5}$ & $\mathbf{3 - 4}$ & $\mathbf{3 - 5}$ & $\mathbf{4 - 5}$ \\
\hline $\mathrm{tm}$ & $2,15^{(1)}$ & $1,88^{(1)}$ & $1,49^{(2)}$ & $1,46^{(2)}$ & 0,04 & $1,55^{(2)}$ & $1,59^{(2)}$ & $1,38^{(2)}$ & $1,43^{(2)}$ & 0,01 \\
$\mathrm{t} \mathrm{tm}$ & $5,82^{(3)}$ & $2,91^{(4)}$ & $9,22^{(4)}$ & $4,36^{(3)}$ & 0,24 & $1,37^{(2)}$ & $1,18^{(2)}$ & $1,53^{(2)}$ & $1,9^{(1)}$ & 0,39 \\
\hline
\end{tabular}

N.B. : (1) différence significative à $20 \%$

(2) différence significative à $30 \%$

(3) différence significative à $5 \%$

(4) différence significative à $10 \%$ 
La répartition horizontale du zooplancton suit une évolution quasi-similaire à celle du phytoplancton, avec un pic d'abondance bien marqué au niveau de la station S3. Mais les modes de répartition des Rotifères et des différentes classes de taille des Crustacés diffèrent les uns des autres:

- le gradient longitudinal décroissant des Rotifères pourrait être relié à celui des matières en suspension. Selon ZUREK (1982), les petits Rotifères à régime microfiltreur sont stimulés par les taux élevées de matières en suspension. Par ailleurs, les Rotifères dotés généralement d'un temps de génération relativement réduit comparés aux Cladocères ou aux Copépodes, seraient favorisés dans les stations en amont où le temps de séjour des eaux est relativement plus court que celui des stations pélagiques (BENZEKRI, 1992). Le relai amont-aval des Rotifères par les Cladocères peut se trouver également renforcé par les phénomènes de compétition entre ces deux groupes (GILBERT et STEMBERGER, 1985 ; GILBERT 1987 et 1989) et par la prédation invertébrée, en particulier celle d'A. robustus, dont le régime est carnivore au stade adulte (FRYER, 1985) ;

- la rareté des Cladocères et des Copépodes en amont pourrait être en outre favorisée et entretenue par les empoissonnements en Carpes chinoises, lesquelles tendent à se cantonner dans les eaux chargées en microphytoplancton de la partie amont. Ce qui serait à l'origine d'une forte prédation en amont, ciblée essentiellement sur les stades adultes de grande taille. À cela, s'ajoute la mauvaise qualité de la nourriture algale dans les stations S2 et S3 en période d'étiage (blooms à Microcystis), facteur qui pourrait accentuer la raréfaction des Crustacés adultes dans la zone amont. En effet, la toxicité de cette algue fait qu'elle est très peu ingérée par le zooplancton herbivore (ZHEN-RONG et al., 1992 ; CHRISTOFFERSEN, 1996 ; ENRISKEN et MOESTRYP, 1997). La faible fécondité des Crustacés en amont, en particulier en saison d'été, serait une conséquence directe du caractère toxique de l'algue (figure 8) ;

- la désertion de la station S1 par le zooplancton serait due à la forte agitation et à la turbidité élevée de cette zone d'embouchure qui empêchent la prolifération du phytoplancton et du zooplancton (PATALAS et SALKI, 1984 ; BRESSAC, 1985 ; FAHDE, 1994). À cela, s'ajoute la faible oxygénation de la station en rapport avec le taux élevé de matières organiques apportées par l'oued Beht (tableau 1) : ces facteurs également propres à inhiber le développement du zooplancton en général (RINGELBERG, 1969 ; GERARD, 1970 ; TAYLOR, 1984).

Comme pour le phytoplancton, ces observations permettent de distinguer 3 zones de la retenue : la zone d'embouchure (S1) désertée par le zooplancton, la zone de transition (S2-S3) où coexistent plus ou moins bien Rotifères et Crustacés, et la zone lacustre (S4 et S5) principalement colonisée par les stades adultes des crustacés. La comparaison des biomasses zooplanctoniques moyennes dans les 5 stations par le test statistique de Student confirme ces résultats (tableau 5).

La répartition longitudinale des peuplements planctoniques dans la retenue El Kansera est cohérente avec celles rapportées dans la littérature, en particulier pour le lac Le Roux (HART, 1990) et le lac Kariba (BEGG, 1971 ; MAGADZA, 1980). Nos résultats semblent par ailleurs en partie en accord avec le modèle 
de MARZOLF (1984), selon lequel la zone amont d'un lac-réservoir est la plus enrichie en peuplements algaux et la zone médiane la plus peuplée en zooplancton. La différence majeure au niveau de la retenue El Kansera se trouve dans la zone d'embouchure (S1), qui est la moins riche en phytoplancton. La forte turbidité de cette zone, en rapport avec la nature déboisée du bassin versant (climat semi-aride), limite le développement algal. Ce qui est au contraire de ce qui se produit pour les lacs-réservoirs situés sous climat tempéré (auxquels le modèle fait référence) et où les apports allochtones sont d'origine organique et stimulent le phytoplancton à l'entrée de la retenue. II serait donc souhaitable d'établir un modèle pour les lacs-réservoirs marocains, voire même africains, et qui tienne compte du rôle de la turbidité dans la répartition horizontale du plancton en général.

\section{5 - CONCLUSIONS}

La répartition longitudinale du phytoplancton et du zooplancton dans la retenue El Kansera apparaît comme une réponse directe aux apports allochtones de l'oued Beht. La quantité très importante de nutriments, surtout phosphorés, apportés par cet oued dans le lac stimule la réponse algale au niveau de la zone de transition entre le milieu lotique et le milieu lacustre. L'excédent de phosphore est stocké dans le sédiment qui constitue un " réservoir " potentiel de cet élément, prêt à le relarguer dés que les conditions du milieu sont favorables (DERRAZ, 1995). Dans la mesure où la fécondité du macrozooplancton chute dans les aires de forte concentration en $M$. aeruginosa, cette Cyanobactérie au caractère toxique reconnu, favorise indirectement le développement d'espèces de petites tailles (Rotifères et petits Crustacés) qui entrent alors en compétition avec les grands filtreurs zooplanctoniques. À ce phénomène, se surimposent les effets de la prédation vertébrée : la présence en amont des populations de Carpe Chinoise concourant également à la raréfaction des grands Crustacés dans cette région. Les effets de $M$. aeruginosa se font au contraire moins sentir en zone pélagique ; sauf sous conditions de sécheresse prolongée où cette espèce colonise l'ensemble de la retenue, en formant des tapis étalés sur plusieurs hectares (DERRAZ, 1995 ; FQIH BERRADA et al., 1999 (1)) ; ce qui entraîne le déclin de la communauté zooplanctonique en cette période (FQIH BERRADA et al., 1999 (2) sous presse). La faible pression de broutage de ces blooms algaux oriente ainsi la chaîne trophique du lac en direction des détritus, formant une grosse réserve de matière organique. Selon AMBLARD (1986) et MARVALIN (1989), une très grande fraction de cette biomasse algale est même dégradée et minéralisée par les bactéries avant d'atteindre le fond. Or, le recyclage des nutriments en surface appuie le prolongement de la période de haute production primaire et accentue ainsi les symptômes de l'eutrophisation du lac.

Ces considérations montrent la nécessité d'intervention pour la sauvegarde de la qualité des eaux de cette retenue, destinées entre autres à l'alimentation en eau potable de deux villes marocaines (Khemisset et Tiflet). La situation est d'autant plus inquiétante de nos jours, où le Maroc est de plus en plus exposé 
à des périodes de sécheresse prolongées. Des moyens de lutte contre la prolifération de la Cyanobactérie $M$. aeruginosa devraient être effectués, avec notamment un contrôle en amont des apports en phosphore et plus particulièrement dans l'oued D'Kor, affluent de l'oued Beht, qui véhicule les eaux usées de Khemisset et qui est très eutrophisant du point de vue phosphore (DERRAZ, 1995).

\section{REMERCIEMENTS}

Ce travail a été réalisé dans le cadre d'une action intégrée entre la faculté des Sciences Aïn Chock de Casablanca (laboratoire biologie et santé), et le laboratoire public d'essais et d'études (Centre de recherche et d'études sur la pollution de l'eau et de l'air, CEREP). Nous tenons à remercier personnellement le Docteur Jabry, directeur du CEREP, qui nous a accueilli dans ce laboratoire, et le Professeur Loudiki de la Faculté des sciences de Marrakech qui nous a aidé à confirmer les espèces algales.

\section{RÉFÉRENCES BIBLIOGRAPHIQUES}

Abdallaoui A., Derraz M., Benabdallah M.Z., et Lek S. (1998). Contribution à l'étude de la relation entre les différentes formes du phosphore dans les sédiments d'une retenue de barrage eutrophe en climat méditerranéen (El Kansera, Maroc). Rev. Sci. Eau, 101-116.

AFNOR, 1994. Recueil des normes françaises, eaux, méthodes d'essais, $342 \mathrm{p}$.

AMBLARD C., 1986. Les nucléotides adénilyques : intérêts pour l'étude de la biomasse, de l'activité métabolique, et de la structure des peuplements phytoplanctoniques lacustres. Th. Doct. d'État, Univ. Clermont II, $317 \mathrm{p}$.

BEGG G.W., 1971. Notes on planktonic fauna of lake Kariba from net collections made in 1967. Lake Kariba Fisheries Research Institute, Unpiblished progress report, 1-35.

BENZEKRI M.A., 1992. Qualité des eaux du lac-réservoir Hassan I $^{\text {er }}$ (Maroc) : hydrochimie et dynamique pluriannuelle du zooplancton. Th. Doct. Fac. Marrakech, $188 \mathrm{p}$.
BOTTRELL H.H. DUCAN A., GLIWICZ Z.M., GRYGIEREK E., HERZIG A., HILLBRICHT-ILKOWSKA A., KURASAWA H., LARSSON P., WEGLENSKA T., 1976. A review of some problems in zooplankton production studies. Norw.J. zool., 24, 419-456.

BRESSAC Y., 1985. Les retenues du Sud Est de la France, influences des arrivées d'eaux et de la qualité du seston sur la structure spatiale du peuplement. Th. Doct. Univ. Provence, $144 \mathrm{p}$.

CARMACK E.C., GRAY, C.R.J., 1982. Patterns of circulation and nutrient supply in a medium residence-time reservoir, Kootenay Lake, British Columbia. Can. Wat. Res. J, 7, 51-69.

CHRISTOFFERSEN K., 1996. Effect of microcystin on growth of single species and on mixed natural populations of heterotrophic nanoflagellates. Nat. Toxins, 4(5), 215-220.

DAFIR J.E., 1997. Contribution à l'étude de quatre écosystèmes marocains différents : la baie de Nador, l'estuaire de Khouribga et les retenues Al Massira et Daourat - 
Échanges eau-sédiment. Th. Doct. Univ. Hassan / Casablanca, 630 p.

DAGG M., 1977. Some effects of patchy food environments on copepods. Limnology and Oceanography, 22, 99-107.

DERRAZ M., 1995. Étude de l'eutrophisation de la retenue de barrage El Kansera (Maroc. Caractéristiques physicochimiques, biodisponibilité du phosphate sédimentaire, écophysiologie de Microcystis et relation des blooms avec les paramètres environnementaux. Th. Univ. Meknès, $120 \mathrm{p}$.

DIAMOND J. M., 1979. Community structure: is it random or is it shaped by species differences and competition? In: R.M. Anderson, B.D. Turner and L.R. Taylor (eds.) Population dynamics. Blackwell, Oxford, England, pp. 165-181.

DRENNER R.W., MC COMAS S.R., 1980. The role of zooplankton escape ability and fish selective feeding and impact of planktivorous fish. In: Evolution and ecology of zooplankton communities. W.C. Kerfoot (ed.). Univ. Press of New England, pp. 587-593.

DUMONT H.J., VAN DE VELDE I., DUMONT S., 1975. The dry weight estimate of biomass in a selection of Cladocera, Copepoda and Rotifera from the plankton, periphyton and benthos of continental waters. Ecologia, 19, 75-97.

ENRISKEN H.P., MOESTRYP O., 1997. Seasonal variation in microcystin contents of Danish Cyanobacteria. Nat. Toxins, 5(3), 99-106.

FAHDE A.; 1994. Étude des peuplements de Cladocères et de Rotifères dans le lac de barrage Al Massira (Maroc), hétérogénéité horizontale, dynamique des populations et broutage. Th. Doct. Univ. Hassan II, Casablanca, 226 pp.

FQIH BERRADA D., BERRADA R., BENZEKRI M.A., (1), 1999. Dynamique du phytoplancton en relation avec certains paramètres physicochimiques dans la retenue El Kansera (Maroc. Annls. Limnology, 35(3), 155-166.

FQIH BERRADA D., BERRADA R., BENZEKRI M.A., FAHDE A., (2) ,1999. Impact des blooms à Microcystis sur le fonctionnement et la structure du zooplancton dans la retenue El Kansera (Maroc. Les Cahiers de la Recherche (sous presse).
FRYER G., 1985. Crustacean diversity in relation to the size of water bodies; some facts and problems. Freshwat. Biol., 15, 361-374.

GEORGE D.G., EDWARDS R.W., 1976. The effect of wind on the distribution of chlorophyll-a and crustacean plankton in a shallow eutrophic reservoir. Journal of Applied Ecology, 13, 667-690.

GERARD G., 1970. Modèles de répartition spatiale en écologie animale. Biométriepraximétrie, 11, 124-190.

GILBERT J.J., 1987. The Polyarthra escape from response: Defense against interference from Daphnia. Hydrobiologia, 147, 235-238.

GILBERT J.J., 1989. The effect of Daphnia interference on a natural rotifer and ciliate community: Short-term bottle experiments. Limnol. Oceanogr., 34(3), 606617.

GILBERT J.J., Stemberger R.S., 1985. Prey capture in the Rotifer Asplanchna girodi. Verh. Internat. Verein. Limnol., 22, 29973000 .

GOLTERMAN H.L., 1973. Physiological limnology an approch to the physiology of lake ecosystems. Elsevier Amsterdam, $498 \mathrm{pp}$.

HART R.C., 1990. Zooplankton distribution in relation to turbidity and related environmental gradients in a large subtropical reservoir: patterns and implications. Freshwat. Biology, 24, 241-263.

JAMES W.F., KENNEDY R.K., MONTGOMERY R.H., NIX J., 1987. Seasonal and longitudinal variations in apparent deposition rates within an Arkansas reservoir. Limnol. Oceanogr. 32, 1169-1176.

KERFOOT W.C., 1975. The divergence of adjacent populations. Ecology, 56, 1298-1313.

KÖHLER J., 1992. Influence of turbulent mixing on growth and primary production of Microcystis aeruginosa in the hypertrophic bautzen reservoir. Arch. Hydrobiol., 123(4), 413-429.

KWIK J.C., CARTER J.C.H., 1975. Populations dynamics of limnetic Cladocera in a beaver pond. J. Fish. Res. Board. Can., 32, 341-346.

LAIR S., 1975. Rôle du zooplancton dans les réseaux trophiques de deux lacs du 
massif central français. Th. Doct. Univ. Clermont-Ferrand, $166 \mathrm{p}$.

LAMOTTE M., BOURLIÈRE F., 1971. Problèmes d'écologie : l'échantillonnage des peuplements animaux des milieux aquatiques. Masson et Cie, Paris.

LE COHU R., 1974. Recherches expérimentales sur l'écologie des algues d'eaux douces : utilisation des enceintes en milieu naturel. Th. Doct. Univ. Rennes, $271 \mathrm{p}$.

LEGENDRE L., WATT W.D., 1972. On a rapid technic for plankton enumeration. Ann. Inst. Oceanogr., Paris, 8, 173-177.

LEVIN L.A., 1984. Life history and dispersal patterns in a dense infaunal polychaeta assemblage: community structure and response to disturbance. Ecology, 65, 1185-1200.

LOHMANN H., 1908. Untersuchungen zur festellung des Vollstandigen gehaltes des Meeres an Plankton. Wiss Meeresunters, Abt. Kiet. N. L., 10, 132-170.

LORENZEN C.J., 1967. Determination of chlorophyll and pheopigments : spectrophotometric equations. Limnol. Oceanogr., 12, 343-346.

LOUDIKI M., 1990. Étude limnologique d'un hydrosystème récemment aménagé dans la région de Marrakech (Maroc). Apports du bassin versant, hydrochimie, algues et macrophytes. Th. Doct. Univ. Saint-Jérôme, $353 \mathrm{p}$.

MAGADZA C.H.D., 1980. The distribution of zooplankton in the Sanyati Bay, Lake Kariba; a multivariable analysis. Hydrobiologia, 70, 57-67.

MALKI, M., 1994. Étude de la communauté phytoplanctonique et des caractéristiques physicochimiques des eaux du lac-réservoir Al Massira. Th. Doct. Univ. Casablanca, $288 \mathrm{p}$.

MARVALIN O., 1989. Abondance, biomasse, activité et relations trophiques des communautés bactériennes hétérotrophes du lac d'Aydat (Puy de Dôme). Contribution à l'étude du fonctionnement des systèmes aquatiques. Th. Doct. Univ. Blaise Pascal Clermont II, $135 \mathrm{p}$.

MARZOLF G.R., 1984. Reservoirs in the great plains of North America. In ECOsystems of the world, 23 lakes and reservoirs. Ed. F.B. Taub., pp. 291-302, Elsevier.
MATTHEW F.K., JOHN R.J., 1989. Summer distribution of nutrients, phytoplankton and dissolved oxygen in relation to hydrology in Table Rock Lake, a large midwestern reservoir. Arch. Hydrobiol., 83(2), 197-225.

ONEP, 1996. Rapport sur la lutte contre l'eutrophisation des lacs-réservoirs marocains, Maroc, Rapport Scientifique $\mathrm{n}^{\circ} 20$, $16 \mathrm{p}$.

PATALAS K., SALKI A., 1984. Effects of impoudments and diversion on the crustacean plankton of southern India lake. Can. J. Fish. Aquat. Sc., 41(4), 613-637.

PIERSEN D.C., WEYHENMEYEr G.A., 1994. High resolution measurements of sediment resuspension above an accumulation bottom in a stratified lake. Hydrobiologia, 284, 43-57.

PINEL-ALLOUL B. METHOT G., VIGNEAULT Y., 1989. Phytoplankton in Quebec lakes: Variations with lake morphometry and with natural anthropogenic acidification. Can. J. Fish. Aquat. Sci., 47, 1047-1056.

PONT D., 1983. Recherches qualitatives sur le peuplement de Copépodes, Cladocères et Ostracodes des rizières de Camargue. Th. Doct. Univ. Provence, Marseille, $353 \mathrm{p}$.

REYNOLDS C.S., 1984. Phytoplankton periodicity: the interactions of form, function and environmental variability. Freshw. Biol., 14(2), 111-142.

RFINIKAINEN M., WALLS M., KEIOLA M., 1994. Acute toxicity of the cyanobacterium Microcystis aeruginosa (strain PCC 7820 ) to Daphnia pulex (Cladocera). Algological studies, 75, 229-237.

RINGELBERG J., 1969. Spatial orientation of planktonic crustaceans. 2. The swimming behaviour in a verical plane. Verh. Internat. Verein. Limnol., 17, 84†- 847.

RODIER J., 1984. L'analyse de l'eau. $7^{\mathrm{e}}$ édition, Dunod, Paris.

TAYLOR L.R., 1984. Assessing and interpreting the special distributions of insect populations. Ann. Rev. Entomol., 29, 321-357.

THÉBAULT L., LESNE J., BOUTIN J.P., 1995. Les Cyanobactéries, leurs toxines et les risques pour la santé. Medecine trop., 55, 375-380. 
THORNTON K.W., KENNEDY R.H., MORGAn A.D., SAUL G.E., 1982. Reservoir water quality sampling design. Water Res. Bull., 18, 471-480.

URABE J., 1989. Relative importance of temporal and spatial heterogeneity in the zooplankton community of an artificial reservoir. Hydrobiologia, 184, 1-6.

VARIS O., 1993. Cyanobacteria dynamics in a restored Finnish lake: a long term simulation study, Hydrobiologia, 268, 129-145.
ZHEN-RONG H., MINJUAN Y., JIAWAN H., 1992. Toxins from Microcystis aeruginosa strain M. 8641. Biochem. J., 9(2), 168-172.

ZOHARY T., ROBART R.D., 1989. Diurnal mixed layers and long-term dominance of Microcystis aeruginosa. J. Plankton. Res., 11(1), 25-48.

ZUREK R., 1982. Effect of suspended materials on zooplankton. Laboratory investigations on Daphnia hyalina Leydig. Acta Hydrobiol., 24, 233-251. 